\title{
Endocannabinoid Signaling within the Basolateral Amygdala Integrates Multiple Stress Hormone Effects on Memory Consolidation
}

\author{
Piray Atsak ${ }^{*}, 1,2,6$, Daniela Hauer ${ }^{1,2,3,6}$, Patrizia Campolongo ${ }^{4}$, Gustav Schelling ${ }^{3}$, Raquel V Fornari ${ }^{5}$ and \\ Benno Roozendaal ${ }^{1,2}$ \\ 'Department of Cognitive Neuroscience, Radboud University Medical Centre, Nijmegen, The Netherlands; ${ }^{2}$ Donders Institute for Brain, Cognition \\ and Behaviour, Radboud University Nijmegen, Nijmegen, The Netherlands; ${ }^{3}$ Department of Anaesthesiology, Ludwig-Maximilians University, \\ Munich, Germany; ${ }^{4}$ Department of Physiology and Pharmacology, Sapienza University of Rome, Rome, Italy; ${ }^{5}$ Núcleo de Cognição e Sistemas \\ Complexos, Centro de Matemática, Computação e Cognição, Universidade Federal do ABC, São Bernardo do Campo, Brazil
}

\begin{abstract}
Glucocorticoid hormones are known to act synergistically with other stress-activated neuromodulatory systems, such as norepinephrine and corticotropin-releasing factor (CRF), within the basolateral complex of the amygdala (BLA) to induce optimal strengthening of the consolidation of long-term memory of emotionally arousing experiences. However, as the onset of these glucocorticoid actions appear often too rapid to be explained by genomic regulation, the neurobiological mechanism of how glucocorticoids could modify the memory-enhancing properties of norepinephrine and CRF remained elusive. Here, we show that the endocannabinoid system, a rapidly activated retrograde messenger system, is a primary route mediating the actions of glucocorticoids, via a glucocorticoid receptor on the cell surface, on BLA neural plasticity and memory consolidation. Furthermore, glucocorticoids recruit downstream endocannabinoid activity within the BLA to interact with both the norepinephrine and CRF systems in enhancing memory consolidation. These findings have important implications for understanding the fine-tuned crosstalk between multiple stress hormone systems in the coordination of (mal)adaptive stress and emotional arousal effects on neural plasticity and memory consolidation.
\end{abstract}

Neuropsychopharmacology (20I5) 40, I485-1494; doi: I0.1038/npp.2014.334; published online 21 January 2015

\section{INTRODUCTION}

Emotional enhancement of memory, our ability to create strong and vivid recollection of biologically significant experiences, is a highly adaptive and fundamental survival mechanism (Christianson, 1992), yet it also contributes to the development of stress-related psychopathologies (de Quervain et al, 2009). Glucocorticoid hormones (corticosterone in rodents, cortisol in humans) are essentially involved in strengthening the consolidation of longterm memory of emotionally arousing experiences in animals (de Kloet et al, 1999; Roozendaal and McGaugh, 2011) and humans (Buchanan and Lovallo, 2001; Kuhlmann and Wolf, 2006). Compelling evidence indicates that glucocorticoids interact with other stress-activated neuromodulatory mechanisms within the basolateral complex of the amygdala (BLA) in influencing neural plasticity and information storage processes (Roozendaal and McGaugh, 2011).

*Correspondence: Dr P Atsak, Department of Cognitive Neuroscience, Radboud University Medical Centre, Geert-Grooteplein Noord 21, Nijmegen, 6500HB, The Netherlands, Tel: +31 24 3610378, Fax: +31 24 36|4214, E-mail: piray.atsak@radboudumc.nl ${ }^{6}$ These authors contributed equally to this work.

Received 9 November 2014; revised 3 December 2014; accepted 18 December 20।4; accepted article preview online 30 December 2014
Most notably, there is extensive support for dynamic and time-controlled interactions between glucocorticoids and arousal-associated noradrenergic activity (Joëls et al, 2011; Roozendaal et al, 2006a). For instance, corticosterone administered after an emotionally arousing training experience rapidly elevates norepinephrine levels in the amygdala (McReynolds et al, 2010) and augments norepinephrineinduced activation of the transcription factor cAMP response element-binding (CREB) protein (Roozendaal et al, 2006b), whereas a blockade of $\beta$-adrenoceptors in the BLA prevents glucocorticoid effects on memory consolidation (Roozendaal et al, 2002). Glucocorticoid signaling within the BLA is also necessary to enable corticotropinreleasing factor (CRF) effects on memory consolidation (Roozendaal et al, 2008). This fine-tuned scheme of stress hormone interactions results in optimal functioning of the BLA, ensuring that stress-related information is retained very well (de Quervain et al, 2009; Kuhlmann and Wolf, 2006; Roozendaal et al, 2006a, 2008). Despite its important functional implications, the neural mechanism(s) of how glucocorticoids rapidly interact with other stress-activated modulatory systems in promoting memory consolidation remain largely elusive.

It has long been recognized that steroid hormones primarily exert their effects on neuronal function by their 
ability to change gene transcription in the nucleus (de Kloet et al, 1999); however, an array of glucocorticoid effects occur in a fashion that cannot be explained by genomic regulation (Atsak et al, 2012b; Dallman, 2005). These findings prompted the hypothesis that glucocorticoids possess membrane-associated receptors through which nongenomic signaling evokes rapid effects on physiology and behavior. The lipid-based endocannabinoid system has recently emerged as one candidate system to mediate such rapid glucocorticoid actions (Atsak et al, 2012a; Evanson et al, 2010; Hill et al, 2010c; Tasker and Herman, 2011). Endocannabinoids, ie, anandamide and 2-arachidonoyl glycerol (2-AG), are short-lived retrograde messengers that are synthesized on demand and released from postsynaptic neurons (Kano et al, 2009). They bind to the cannabinoid receptor subtype 1 ( $\mathrm{CB} 1$ receptor) at presynaptic sites to control neurotransmitter release and excitatory-inhibitory balance in neuronal networks (Kano et al, 2009). CB1 receptors are abundantly expressed in the BLA (and other limbic regions) where they regulate stress and emotional arousal effects on synaptic transmission, neuronal firing, and memory (Akirav, 2011; Campolongo et al, 2009; Pistis et al, 2004). Findings that corticosterone administration rapidly increases amygdala endocannabinoid levels (Hill et al, 2010a), whereas a blockade of CB1 receptor activity in the BLA prevents corticosterone-induced memory enhancement (Campolongo et al, 2009) reiterate the growing notion of the endocannabinoid system as a rapid mediator of responses to stress and stress hormones (Atsak et al, 2012b; Hill and McEwen, 2010b). The current study investigated whether endocannabinoid signaling within the BLA mediates the permissive actions of glucocorticoids onto the noradrenergic and CRF systems and thereby aids in integrating the modulatory influences of multiple stress hormone signals on memory consolidation.

\section{MATERIALS AND METHODS}

\section{Subjects}

Male Sprague-Dawley rats (280-320 g at time of surgery; Charles River, Kisslegg, Germany) were kept individually in a temperature-controlled $\left(22^{\circ} \mathrm{C}\right)$ colony room (07:00-19:00 h lights on) with ad libitum access to food and water. Training and testing were performed between 10:00 and 15:00 h. All procedures were performed in compliance with the European Communities Council Directive of 24 November 1986 (86/609/EEC) and approved by the Institutional Animal Care and Use Committee.

\section{Surgery}

Cannula implantation was performed according to a standardized protocol (Fornari et al, 2012). In brief, the rat was positioned in a stereotaxic frame (Kopf Instruments, Tujunga, CA) and two stainless-steel guide cannulae (15 mm; 23 gauge; Component Supply Co/SKU Solutions, Fort Meade, FL) were implanted bilaterally with the cannula tips $2.0 \mathrm{~mm}$ above the BLA (anteroposterior, $-2.8 \mathrm{~mm}$ from Bregma; mediolateral, $\pm 5.0 \mathrm{~mm}$ from midline; dorsoventral, $-6.5 \mathrm{~mm}$ from skull surface (Paxinos and Watson, 2007)). The rats were allowed to recover for a minimum of 7 days before initiation of training and were handled three times for $1 \mathrm{~min}$ each during this recovery period. See Supplementary Materials and Methods for full protocol.

\section{Inhibitory Avoidance Apparatus and Procedures}

Rats were trained and tested in an inhibitory avoidance apparatus (see Supplementary Materials and Methods). After the rat stepped into the dark compartment, the sliding door was closed and a single inescapable footshock $(0.60 \mathrm{~mA})$ was delivered for $1 \mathrm{~s}$. For the experiment with systemic drug administration, a lower footshock intensity $(0.35 \mathrm{~mA}, 1 \mathrm{~s})$ was used. For 48 -h retention testing, the rat was placed into the starting compartment and the latency to re-enter the shock compartment with all four paws was measured (maximum latency of $600 \mathrm{~s}$ ). Longer latencies indicate better retention.

\section{Drugs and Infusion Procedures}

The GR agonist RU $28362(11 \beta, 17 \beta$-dihydroxy-6,21-dimethyl-17 $\alpha$-pregna-4,6-trien-20yn-3-one; 1, 3, or $10 \mathrm{ng}$; Aventis, Frankfurt, Germany), the membrane-impermeable glucocorticoid Cort:BSA (1, 3, or $10 \mathrm{ng}$; Sigma-Aldrich), the CRF-binding protein inhibitor human/rat $\mathrm{CRF}_{6-33}(0.01,0.1$, or $1 \mu \mathrm{g}$; Bachem, La Jolla, CA), and the $\beta$-adrenoceptor agonist clenbuterol (1, 10, or $100 \mathrm{ng}$; Sigma-Aldrich) were infused into the BLA $(0.2 \mu \mathrm{l}$ per hemisphere $)$ immediately after inhibitory avoidance training either alone or together with the selective CB1 receptor antagonist AM251 ( N-1-(2,4dichlorophenyl)-5-(4-iodophenyl)-4-methyl- $N$-1-piperidinyl$1 \mathrm{H}$-pyrazole-3-carboxamide; $0.14 \mathrm{ng}$; Sigma-Aldrich). The synthetic cannabinoid agonist WIN55,212-2 $(R-(+)-(2,3-$ dihydro-5-methyl-3-[(morpholinyl)methyl]pyrol[1,2,3-de]1,4-benzoxazin-6-yl)(1-naphthalenyl) methanone monomethanesulfonate; 10,30 , or $100 \mathrm{ng}$; Sigma-Aldrich) was infused into the BLA alone or together with the GR antagonist RU 38486 (17 $\beta$-hydroxy-11 $\beta$-(4-dimethylaminophenyl)-17 $\alpha$-(1-propynyl)-oestra-4,9-dien-3-one; 1 ng; Aventis) or $\beta$-adrenoceptor antagonist propranolol $(0.5 \mu \mathrm{g}$; SigmaAldrich). In the last experiment, clenbuterol (1, 10, or $100 \mathrm{ng})$ or $\mathrm{CRF}_{6-33}(1 \mu \mathrm{g})$ was given together with WIN55,212-2 (1 ng) and RU 38486 (1 ng). Each animal was used for a single behavioral experiment and drug administration only. Drug preparation and the infusion procedures are detailed in Supplementary Materials and Methods.

Corticosterone (3 mg/kg; Sigma-Aldrich) either alone or together with the CB1 receptor antagonist SR141716 (rimonabant, 5-(4-chlorophenyl)-1-(2,4-dichloro-phenyl)4-methyl- $\mathrm{N}$-(piperidin-1-yl)-1H-pyrazole-3-carboxamide; $1 \mathrm{mg} / \mathrm{kg}$; Kemprotec Ltd., Middlesbrough, UK) was given subcutaneously, in a volume of $2 \mathrm{ml} / \mathrm{kg}$, immediately after the training. This dose of corticosterone results in an increase in circulating corticosterone levels within the physiological range and enhances memory consolidation (Miranda et al, 2008). The corticosterone and SR141716 mixture was dissolved in 5\% polyethylene glycol, $5 \%$ tween80 , and $5 \%$ ethanol in saline. All drug doses are based on previous studies; see Supplementary Materials and Methods for corresponding references. 


\section{Histology}

Rats were anesthetized with an overdose of sodium pentobarbital and perfused transcardially with $0.9 \%$ saline and $4 \%$ formaldehyde. The brains were removed and processed for verification of cannula placement within the BLA (see Supplementary Materials and Methods). Rats with injection needle placements outside the BLA or with extensive tissue damage at the injection needle tips were excluded from final analysis; the total exclusion rate was $14.5 \%$.

\section{Immunohistochemistry}

Sixty minutes after inhibitory avoidance training and systemic drug treatment, rats were perfused transcardially with ice-cold $4 \%$ paraformaldehyde in $0.1 \mathrm{M}$ phosphate buffer, $\mathrm{pH}$ 7.4. Brains were removed and coronal sections at the level of the BLA were cut at $20 \mu \mathrm{m}$. Free-floating BLA sections were incubated with a monoclonal pCREB (Ser133) antibody (rabbit, Cell Signaling Technology, \#9198, 1:30) alone or together with a CaMKII antibody (mouse anticalcium/calmodulin-dependent protein kinase II, Millipore, 1:500) at $4{ }^{\circ} \mathrm{C}$ for $48 \mathrm{~h}$. Sections treated with the pCREB antibody were incubated with biotinylated donkey antirabbit IgG secondary antibody $(2 \mu \mathrm{g} / \mathrm{ml}$, Jackson ImmunoResearch), followed by incubation with avidin-biotin complex-peroxidase (1:500, ABC kit, Vector laboratories) and stained with $0.025 \%$ diaminobenzidine, $0.2 \%$ nickel ammonium sulfate adding $0.3 \% \mathrm{H}_{2} \mathrm{O}_{2}$. pCREB-positive cells were counted (positive cells $/ 0.1 \mathrm{~mm}^{2}$ ) in the BLA. Sections treated with both pCREB and CAMKII antibodies were incubated with a cocktail of specific fluorochrome-conjugated secondary antibodies (Alexa Fluor 488 donkey antirabbit 1:200 and Alexa Fluor 561 donkey anti-mouse 1:200, Invitrogen). Qualitative images were acquired with a confocal microscope (Leica-microsystems, TCS SP2 AOBS, Mannheim, Germany) (see Supplementary Materials and Methods).

\section{Statistics}

Data are presented as mean \pm SEM. Inhibitory avoidance latencies and pCREB cell counts were analyzed with two- or three-way ANOVAs. Further analysis used Fisher's post hoc tests to determine the source of the detected significances, when appropriate. To determine whether learning had occurred, paired $t$-tests were used to compare training and retention latencies of individual rats. A probability level of $<0.05$ was accepted as statistical significance.

\section{RESULTS}

\section{The Endocannabinoid System of the BLA Mediates Glucocorticoid Effects on Memory Consolidation}

We first determined to what extent endocannabinoid signaling is implicated in regulating glucocorticoid effects per se on memory consolidation. Rats, equipped with bilateral cannulae aimed at the BLA (Figure 1a), were trained on a one-trial inhibitory avoidance task and immediately after the training trial, ie, during the consolidation phase, administered the specific GR agonist RU 28362 either alone or together with the CB1 receptor antagonist AM251. Retention of the training was tested $48 \mathrm{~h}$ later. Average stepthrough latencies during training were $13.4 \pm 0.7 \mathrm{~s}$ (mean \pm SEM) and did not differ between groups (see Supplementary Material for training latencies of all experiments). Fortyeight-hour retention test latencies of rats administered vehicle into the BLA immediately after training were significantly longer than their entrance latencies on the training trial (paired $t$-test: $P=0.01$ ), indicating that the rats retained significant memory of the shock experience. As shown in Figure 1b, two-way ANOVA for retention latencies revealed significant RU $28362\left(F_{3,80}=2.98 ; P=0.04\right)$ and AM251 effects $\left(F_{1,80}=4.26 ; P=0.04\right)$ as well as a significant interaction effect $\left(F_{3,80}=4.07 ; P=0.04\right)$. RU $28362(1,3$ or $10 \mathrm{ng})$ induced dose-dependent retention enhancement (1 ng: $P<0.01)$. AM251 (0.14 ng) alone did not impair retention, but blocked the retention enhancement induced by RU 28362 . Rats treated with AM251 together with RU 28362 ( $1 \mathrm{ng}$ ) had significantly shorter latencies than those given the same dose of RU 28362 alone $(P<0.01)$.

Next, we investigated whether glucocorticoid effects on the endocannabinoid system in regulating memory consolidation involve the activation of a corticosteroid receptor on the cell surface. Emerging evidence indicates that rapid glucocorticoid actions are mediated either by a putative G-protein-coupled membrane receptor or by an isoform of the classical steroid receptor that is located at the membrane (Johnson et al, 2005). The membrane-impermeable glucocorticoid ligand Cort:BSA, ie, corticosterone conjugated to bovine serum albumin, was administered posttraining into the BLA either alone or together with AM251. Two-way ANOVA for 48-h retention latencies revealed significant Cort:BSA $\quad\left(F_{3,92}=2.85 ; \quad P=0.04\right)$ and AM251 effects $\left(F_{1,92}=7.33 ; P=0.008\right)$ as well as a significant interaction effect $\left(F_{3,92}=2.76 ; P=0.046\right)$. Cort:BSA $(1,3$ or $10 \mathrm{ng})$ dosedependently enhanced retention (1 ng: $P<0.05$; 3 ng: $P<0.01$ ), whereas co-administration of the $\mathrm{CB1}$ receptor antagonist AM251 (0.14 ng) blocked the retention enhancement produced by Cort:BSA ( 3 ng: $P<0.05$; Figure 1c).

These findings indicate that $\mathrm{CB} 1$ receptor signaling within the BLA is required for enabling the memory-enhancing effect of GR agonist or Cort:BSA. However, glucocorticoids might strengthen consolidation processes by concurrently activating GRs at several functional levels within the cell or local network, some that are independent of endocannabinoid signaling. To determine the significance of the endocannabinoid pathway in contributing to the memoryenhancing effects of glucocorticoids, we examined whether direct activation of endocannabinoid signaling with posttraining intra-BLA infusions of the synthetic cannabinoid agonist WIN55,212-2 (10, 30, or $100 \mathrm{ng})$ would enhance memory consolidation under a condition where other GRmediated actions are pharmacologically blocked by its specific antagonist RU 38486 (1 ng) (Barsegyan et al, 2010). Two-way ANOVA for 48-h retention latencies revealed a significant WIN55,212-2 effect $\left(F_{3,91}=13.65 ; P<0.0001\right)$, but no GR antagonist effect $\left(F_{1,91}=0.33 ; P=0.57\right)$ or interaction between both drugs $\left(F_{3,91}=0.04 ; P=0.99\right)$. As shown in Figure 1d, WIN55,212-2 administered alone induced dose-dependent retention enhancement (10 ng: $P<0.01)$. As with WIN55,212-2 alone, the $10 \mathrm{ng}$ dose of WIN55,212-2 co-infused with the GR antagonist induced 
a

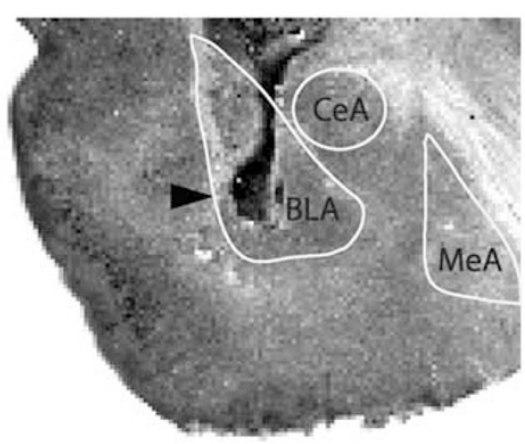

C

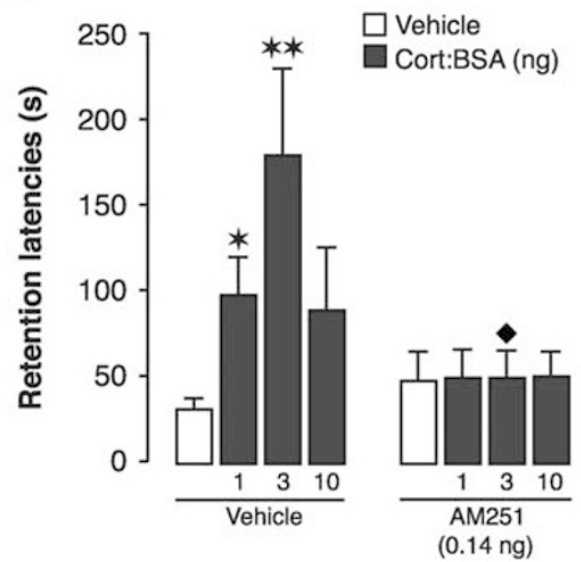

b

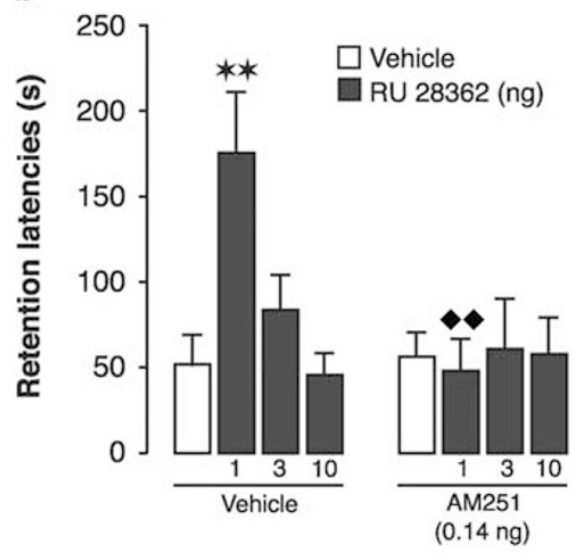

d

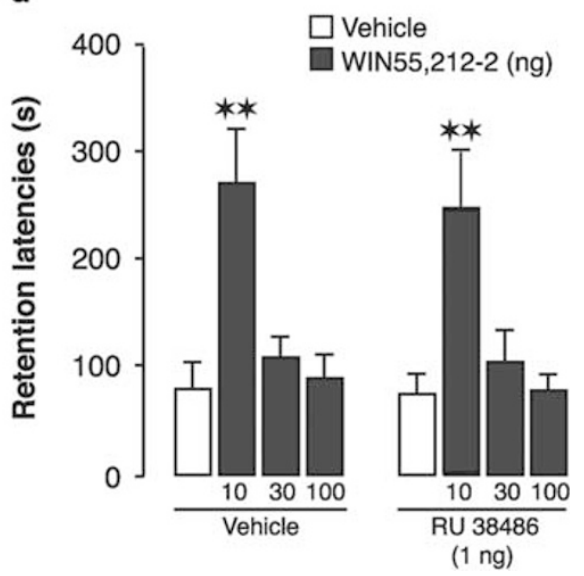

Figure I Glucocorticoids interact with the endocannabinoid system of the BLA in enhancing memory consolidation of inhibitory avoidance training. (a) Representative photomicrograph illustrating placement of a needle tip within the BLA, basolateral amygdala. Arrow points to the needle tip. MeA, medial amygdala; CeA, central amygdala. (b) Retention latencies of rats given posttraining intra-BLA infusions of the GR agonist RU 28362 (I, 3, or I0 ng in $0.2 \mu \mathrm{l})$ alone or together with the CBI receptor antagonist AM25I (0.14 ng). *** $P<0.0$ I vs vehicle; $\leftrightarrow P<0.0$ I vs RU 28362 only $(n=9-12 / g r o u p)$. (c) Retention latencies of rats given posttraining intra-BLA infusions of the membrane-impermeable glucocorticoid Cort:BSA (I, 3, or $\mid 0 \mathrm{ng}$ in $0.2 \mu \mathrm{l})$ alone or together with AM25 I ( $0.14 \mathrm{ng})$. * $P<0.05$; $* * P<0.0$ I vs vehicle; $P<0.05$ vs Cort:BSA alone $(n=||-\mid 4 /$ group). (d) Retention latencies of rats given posttraining intra-BLA infusions of the cannabinoid agonist WIN55,2I2-2 (I0,30, or $100 \mathrm{ng}$ in $0.2 \mu \mathrm{l})$ alone or together with the GR antagonist RU 38486 (I ng). $* * P<0.0$ I vs vehicle; ( $n=10-14 /$ group). Data depict 48-h retention latencies (mean + SEM) in seconds $(s)$.

significantly longer retention latencies $(P<0.01)$. These findings indicate that glucocorticoid-induced recruitment of downstream endocannabinoid signaling is a major route by which glucocorticoids enhance the consolidation of memory, as concurrent blockade of other GR-mediated mechanisms does not seem to prevent or attenuate this memory enhancement.

\section{The Endocannabinoid System Mediates Glucocorticoid Effects on Neural Plasticity within the BLA}

Rapid glucocorticoid actions on memory consolidation are known to involve phosphorylation of the transcription factor CREB, recruitment of co-activators, and changes in chromatin function (Roozendaal et al, 2010). Extensive evidence implicates CREB activation within the BLA as an essential step in the molecular process underlying memory consolidation (Josselyn et al, 2004). To investigate whether endocannabinoid activity is required for mediating glucocorticoid effects on CREB activation, the CB1 receptor antagonist SR141716 (rimonabant; $1 \mathrm{mg} / \mathrm{kg}$ ) was administered subcutaneously together with corticosterone $(3 \mathrm{mg} / \mathrm{kg})$ immediately after inhibitory avoidance training, and the number of cells expressing immunoreactivity for phosphorylated CREB (pCREB) within the BLA was assessed $1 \mathrm{~h}$ later. Two-way ANOVA for the number of pCREB-positive cells revealed significant corticosterone $\left(F_{1,18}=6.21\right.$, $P=0.02)$ and SR141716 effects $\left(F_{1,18}=8.50, P=0.001\right)$ as well as a significant interaction effect $\left(F_{1,18}=6.53, P=0.02\right)$. As shown in Figures $2 a$ and $b_{1}$, corticosterone administration significantly increased the number of pCREB-positive cells $(170 \pm 14 \% ; P<0.01$, relative to vehicle), and coadministration of SR141716 blocked the corticosterone effect $(94 \pm 13 \% ; \quad P<0.01)$. Qualitative double labeling indicated that the majority of pCREB-expressing cells also stained positive for CAMKII, a marker for glutamatergic pyramidal neurons (Figure $2 b_{2}$ ). Separate groups of rats were tested for retention $48 \mathrm{~h}$ after the training and drug treatment. Two-way ANOVA for retention latencies indicated significant corticosterone $\left(F_{1,36}=9.09 ; P=0.005\right)$ and 


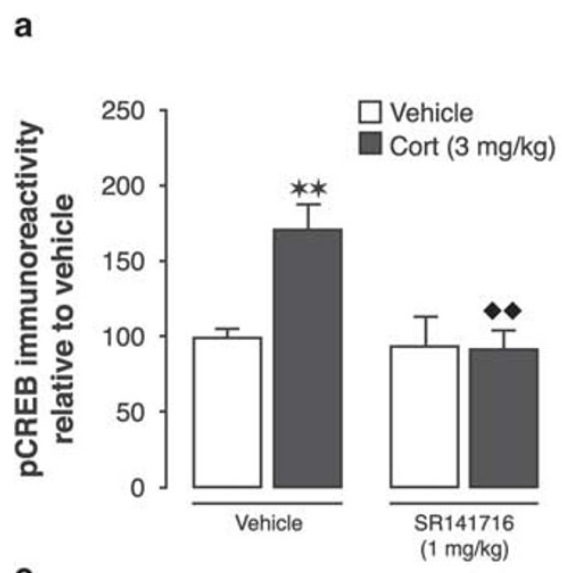

b1
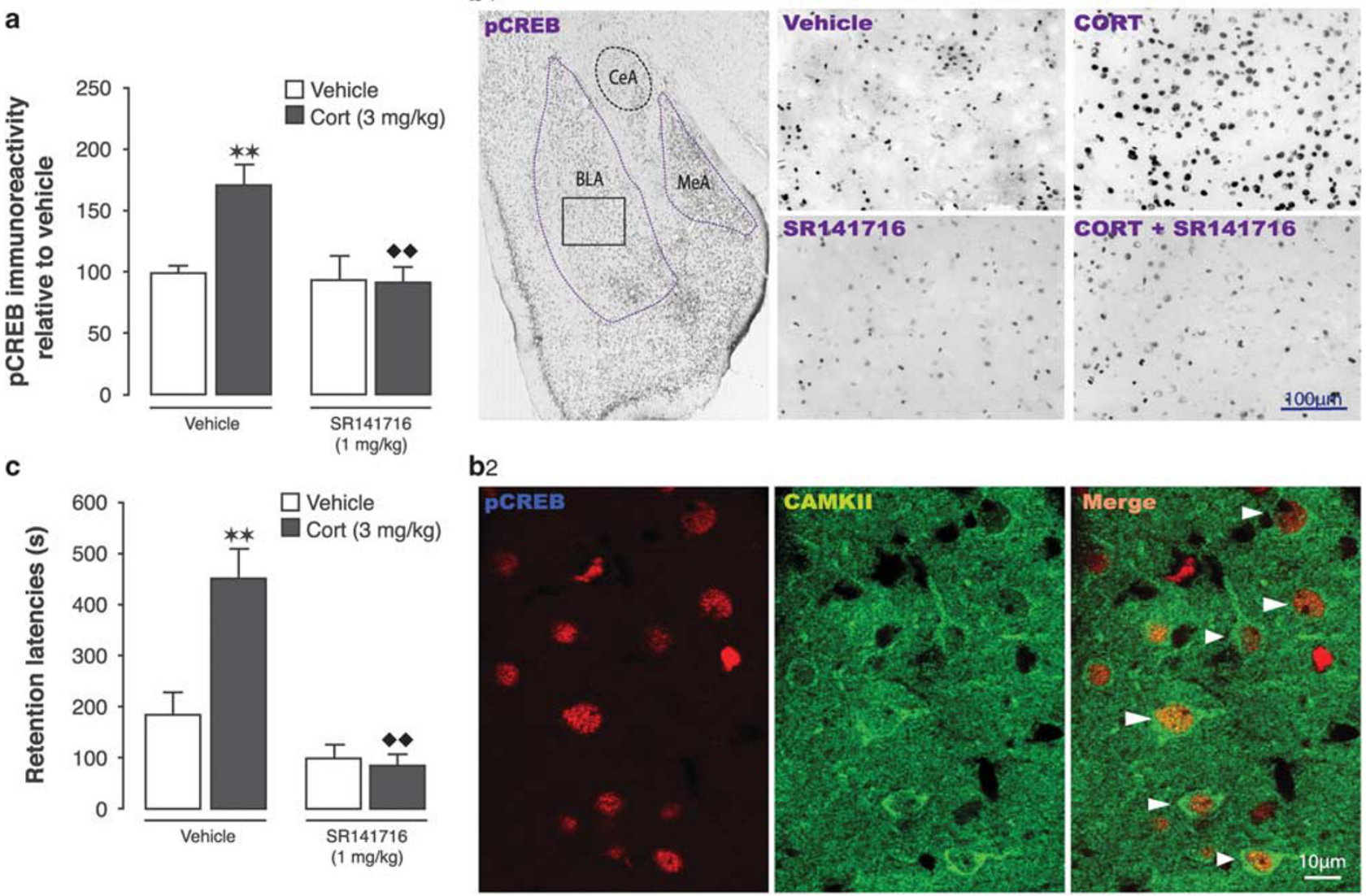

Figure 2 Glucocorticoids require endocannabinoid signaling to induce a training-associated increase in CREB phosphorylation within the BLA. (a) The number of PCREB-positive neurons in the BLA as assessed I h after inhibitory avoidance training followed by a systemic injection of corticosterone (CORT,

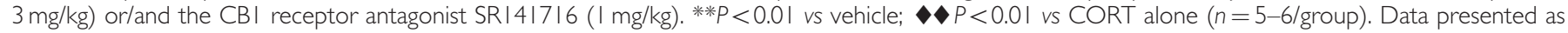
percentages relative to vehicle. (bI) Photomicrographs of PCREB immunostaining within the BLA I h after inhibitory avoidance training and systemic injection of CORT (3 mg/kg), SRI41716 (I mg/kg), or drug combination. BLA, basolateral amygdala, CeA, central amygdala, MeA, medial amygdala. (b2) Confocal images illustrating cells positive for PCREB (left), CAMKII (middle), and double labeling (right) in the BLA. (c) Forty-eight hours retention latencies $($ mean $+\mathrm{SEM})$ in seconds $(\mathrm{s})$ of rats given immediate posttraining systemic injection of CORT $(3 \mathrm{mg} / \mathrm{kg})$ alone or together with SRI4I7/6 (I mg/kg). $* * P<0.0$ I vs vehicle; $\diamond P<0.0$ I vs corticosterone alone ( $n=8-$ I0/group).

SR141716 effects $\left(F_{1,36}=27.17 ; P<0.0001\right)$ as well as a significant interaction $\left(F_{1,36}=10.98 ; P=0.002\right)$. The corticosterone injection enhanced retention performance $(P<0.01)$, whereas co-administration of SR141716 blocked the corticosterone effect $(P<0.01$; Figure $2 \mathrm{c})$.

\section{The Endocannabinoid System Mediates Glucocorticoid Actions on the Noradrenergic System in Regulating Memory Consolidation}

Extensive evidence indicates that glucocorticoids interact with arousal-associated noradrenergic activation within the BLA to selectively enhance the consolidation of memory of emotionally salient experiences (Roozendaal et al, 2006a, 2006b). Previously we showed that the $\beta$-adrenoceptor antagonist propranolol infused into the BLA blocks glucocorticoid effects on memory consolidation (Roozendaal et al, 2006a, 2006b). We investigated whether the memoryenhancing effects of endocannabinoids, like those of glucocorticoids, depend on endogenous noradrenergic activity within the BLA. Rats were trained on the inhibitory avoidance task and received posttraining infusions of propranolol $(0.5 \mu \mathrm{g})$ together with the cannabinoid agonist WIN55,212-2 (10,30, or $100 \mathrm{ng})$. As shown in Figure 3a, two-way ANOVA for 48-h retention latencies revealed significant WIN55,212-2 $\left(F_{3,75}=4.33 ; P=0.007\right)$ and propranolol effects $\left(F_{1,75}=4.64 ; P=0.03\right)$ as well as a significant interaction between conditions $\left(F_{3,75}=3.15 ; P=0.03\right)$. WIN55,212-2 alone induced dose-dependent retention enhancement (10 ng: $P<0.01)$. Propranolol did not impair retention, but blocked the retention enhancement induced by concurrently administered WIN55,212-2 (10 ng: $P<0.01)$.

Previously, we reported that GR activation in the BLA might facilitate memory consolidation via a rapid potentiation of the norepinephrine-signaling cascade through an interaction with $G$ protein-mediated effects (Roozendaal et al, 2002). We found that a suppression of GR signaling with its specific antagonist RU 38486 reduces the sensitivity of the BLA to the memory-enhancing effects of noradrenergic stimulation such that a much higher dose of the $\beta$-adrenoceptor agonist clenbuterol is required to induce memory enhancement. If the endocannabinoid system is the primary route by which glucocorticoids alter the sensitivity 
a

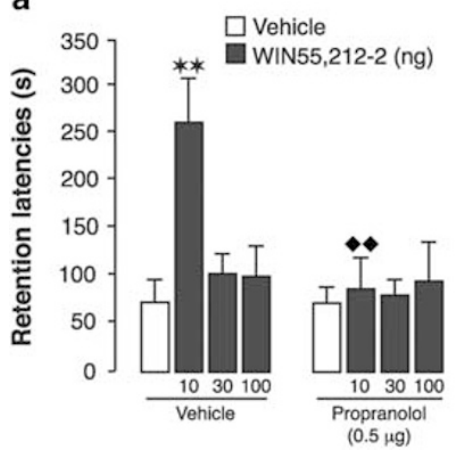

b

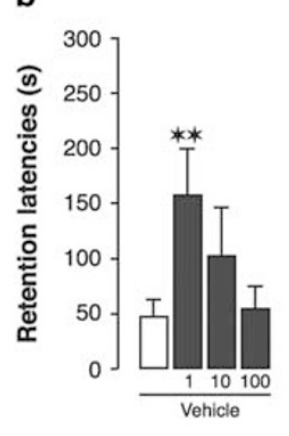

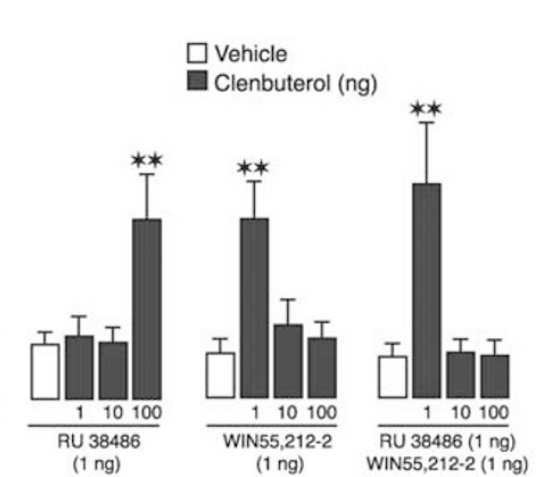

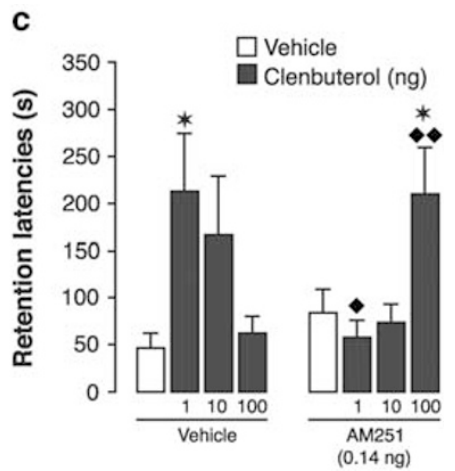

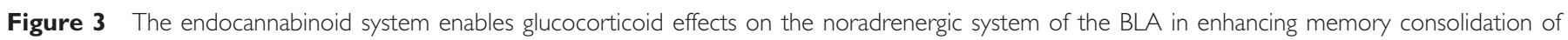

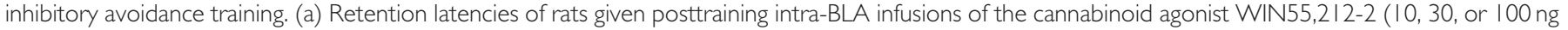

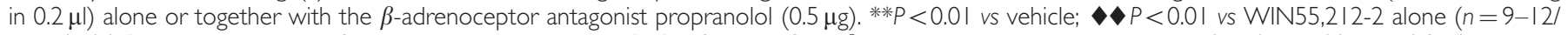

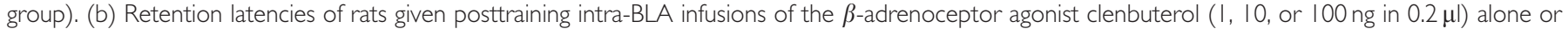

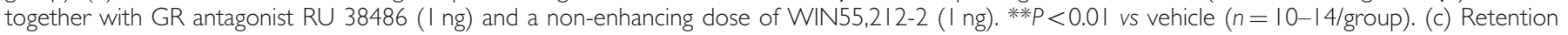

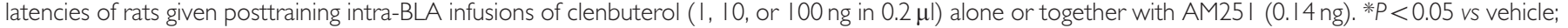
$\diamond P<0.05 ; \diamond P<0.01$ vs clenbuterol alone $(n=9-12$ /group). Data depict 48-h retention latencies (mean + SEM) in seconds(s).

of the BLA to clenbuterol, then pharmacological augmentation of cannabinoid activity with an otherwise non-enhancing dose of WIN55,212-2 should compensate for this GRblockade effect. On the other hand, if the glucocorticoid effect depends on multiple concurrent mechanisms, elevating cannabinoid signaling should not, or only partly, reverse the GR antagonist effect. As shown in Figure 3b, three-way ANOVA for 48 -h retention latencies revealed a significant clenbuterol effect $\left(F_{3,167}=13.05 ; P<0.0001\right)$ as well as a significant interaction between clenbuterol, GR antagonist, and cannabinoid agonist $\left(F_{3,167}=3.00 ; P=0.03\right)$. When clenbuterol (1, 10, or $100 \mathrm{ng}$ ) was administered alone, the $1 \mathrm{ng}$ dose enhanced retention latencies $(P<0.01)$, whereas the 10 and $100 \mathrm{ng}$ doses were ineffective. As expected, the GR antagonist RU 38486 (1 ng) shifted the dose-response effects of clenbuterol such that a 100 times higher dose of clenbuterol was required to induce memory enhancement (100 ng: $P<0.01)$. Most importantly, whereas the low dose of WIN55,212-2 (1 ng) alone was insufficient to enhance memory or alter the dose-response effects of clenbuterol, it totally prevented the dose-shift induced by the GR antagonist ( $1 \mathrm{ng}: P<0.01$ ). These findings indicating that augmentation of cannabinoid signaling fully compensates for the lack of GR activation and normalizes the sensitivity of the BLA to noradrenergic stimulation show that the endocannabinoid pathway is the primary route by which glucocorticoids alter the memory-enhancing effects of noradrenergic stimulation.

If the endocannabinoid system mediates the glucocorticoid influence on the noradrenergic system, then a blockade of $\mathrm{CB} 1$ receptors with posttraining intra-BLA infusions of AM251 should produce an identical shift in the doseresponse effects of clenbuterol. As shown in Figure 3c, two-way ANOVA for 48 -h retention latencies revealed a significant interaction between the two drug conditions $\left(F_{3,75}=6.13 ; P=0.0009\right)$. The lowest dose of clenbuterol $(1 \mathrm{ng})$ alone enhanced retention $(P<0.05)$, whereas higher doses (10 or $100 \mathrm{ng}$ ) were ineffective. When AM251 $(0.14 \mathrm{ng})$ was co-infused with clenbuterol, the lowest dose of clenbuterol failed to enhance retention performance, and the $100 \mathrm{ng}$ dose of clenbuterol was necessary to induce significant retention enhancement $(P<0.01)$. Thus, highly comparable to the effect of GR antagonism, a blockade of $\mathrm{CB} 1$ receptors reduces the sensitivity of the $\mathrm{BLA}$ to the memory-enhancing effect of noradrenergic stimulation.

\section{Endocannabinoids Mediate Glucocorticoid Effects on the CRF System in Regulating Memory Consolidation}

To investigate whether the endocannabinoid system serves a more general role in regulating permissive glucocorticoid effects on other stress-activated modulatory systems, we examined its involvement in mediating glucocorticoid effects onto the CRF system. Previously, we reported that a suppression of GR signaling in the BLA completely blocked the memory-enhancing effects of $\mathrm{CRF}_{6-33}$, a drug that enhances memory consolidation by displacing CRF from the CRF-binding protein and increasing the 'free' concentration of endogenous CRF (Roozendaal et al, 2008). In analogy to the experiment above, we investigated whether pharmacological activation of $\mathrm{CB} 1$ receptors with a non-enhancing dose of WIN55,212-2 would override the GR-blockade effect on $\mathrm{CRF}_{6-33}$-induced memory enhancement. Three-way ANOVA for 48 -h retention latencies revealed a significant $\mathrm{CRF}_{6-33}$ effect $\left(F_{1,83}=55.13 ; P<0.0001\right)$ and significant interaction between $\mathrm{CRF}_{6-33}, \mathrm{GR}$ antagonist, and cannabinoid agonist $\left(F_{1,83}=5.48 ; P=0.02\right)$. Figure 4 a shows that posttraining intra-BLA infusions of the GR antagonist RU 38486 ( $1 \mathrm{ng}$ ) blocked the memory-enhancing effect of $\mathrm{CRF}_{6-33}(0.1 \mu \mathrm{g} ; \mathrm{P}<0.01)$. Importantly, whereas WIN55,2122 (1 ng) alone did not enhance memory or modified the memory-enhancing effect of $\mathrm{CRF}_{6-33}$, it fully compensated for the GR antagonist effect and enabled $\mathrm{CRF}_{6-33}$ to enhance memory consolidation in the absence of GR signaling $(P<0.01)$.

To further explore the functional commonality of the glucocorticoid and endocannabinoid systems in regulating CRF effects on memory consolidation, we investigated whether $\mathrm{CB} 1$ receptor blockade would also prevent the memory-enhancing effect of $\mathrm{CRF}_{6-33}$. Two-way ANOVA for 48-h retention latencies demonstrated significant $\mathrm{CRF}_{6-33}$ $\left(F_{3,86}=3.42 ; \quad P=0.02\right)$ and AM251 effects $\left(F_{1,86}=4.50\right.$; 
a

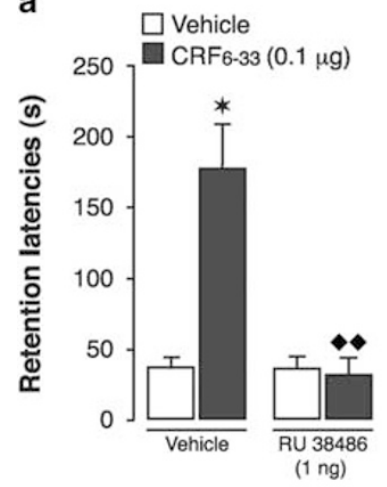

b

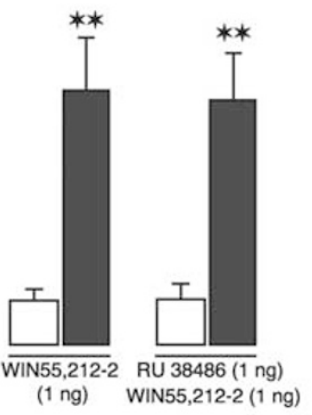

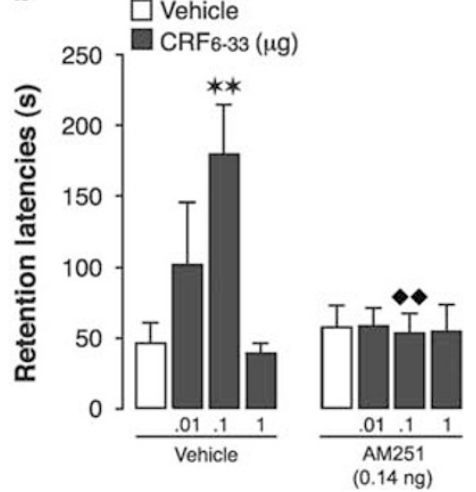

Figure 4 The endocannabinoid system enables glucocorticoid effects on the CRF system of the BLA in enhancing memory consolidation of inhibitory avoidance training. (a) Retention latencies of rats given posttraining intra-BLA infusions of the CRF-binding protein inhibitor $\mathrm{CRF}_{6-33}(0.1 \mu \mathrm{g}$ in $0.2 \mu \mathrm{l})$ alone or together with GR antagonist RU 38486 (I ng) and cannabinoid agonist WIN55,2I2-2 (I ng). $* P<0.0$ I, ** $P<0.0$ I vs vehicle; $\bullet P<0.0$ I vs $C R F_{6-33}$ alone ( $n=9-13$ /group). (b) Retention latencies of rats given posttraining intra-BLA infusions of $\mathrm{CRF}_{6-33}(0.0 \mathrm{I}, 0 . \mathrm{I}$, or I $\mu \mathrm{g}$ in $0.2 \mu \mathrm{l})$ alone or together with the $\mathrm{CBI}$ receptor antagonist $\mathrm{AM} 25 \mathrm{I}$ ( 0.14 ng). ${ }^{*} * \mathrm{P}<0.0$ I vs vehicle; $\bullet P<0.0$ I vs $\mathrm{CRF}_{6-33}$ alone $(n=10-\mid 3 /$ group). Data depict $48-\mathrm{h}$ retention latencies $($ mean $+\mathrm{SEM})$ in seconds $(\mathrm{s})$.

$P=0.04)$ as well as a significant interaction effect $\left(F_{3,86}=3.69 ; P=0.02\right)$. As shown in Figure $4 \mathrm{~b}$, posttraining $\mathrm{CRF}_{6-33}(0.01,0.1$, or $1 \mu \mathrm{g})$ infusions into the BLA induced dose-dependent retention enhancement $(0.1 \mu \mathrm{g}: P<0.01)$, whereas the CB1 receptor antagonist AM251 (0.14 ng) blocked the $\mathrm{CRF}_{6-33}$ effect $(P<0.01)$, indicating that antagonism of either $\mathrm{CB} 1$ receptor or GR results in a comparable and complete inhibition of CRF-induced memory enhancement.

\section{DISCUSSION}

The present study was undertaken to investigate whether the endocannabinoid system of the BLA mediates the permissive actions of glucocorticoid hormones onto the noradrenergic and CRF systems in regulating memory consolidation. The interest of this question stems from previous work, indicating that glucocorticoids selectively enhance the consolidation of memory for emotionally salient experiences via critical interactions with other stress-activated hormonal and neurotransmitter systems (Roozendaal et al, 2006b; Van Stegeren et al, 2007). However, as the onset of these glucocorticoid effects appear too rapid to be mediated via genomic GR pathways, the neurobiological mechanism of how glucocorticoids modify the memory-enhancing properties of these other systems remained to be determined.

Consistent with the findings of many previous pharmacological studies on memory, the GR agonist RU 28362 and the membrane-impermeable ligand Cort:BSA (as well as the other agonists used) enhanced memory in a dose-dependent manner. The mechanism underlying this dose-response effect is unknown but the generality of such inverted U-shaped effects across drug systems as well as acute stress effects on memory (eg, Roozendaal et al, 2006b; Salehi et al, 2010) strongly argues that the ineffectiveness of higher doses in the present study is not caused by any specific characteristics of the glucocorticoid drugs. Our finding that the CB1 receptor antagonist AM251 administered into the BLA after inhibitory avoidance training blocked the memory-enhancing effect of RU 28362 and Cort:BSA indicates that endocannabinoid signaling is essentially involved in regulating glucocorticoid effects on memory consolidation. Endocannabinoid signaling was also required to mediate glucocorticoid effects on pCREB expression, a well-defined mechanism for neural plasticity (Josselyn et al, 2004; Roozendaal et al, 2006a), in BLA pyramidal neurons after inhibitory avoidance training. This glucocorticoid action most likely involves the activation of a GR on the cell surface (Johnson et al, 2005), launching a G-proteindependent signaling cascade that induces the synthesis of endocannabinoid ligands ( $\mathrm{Di}$ et al, 2003; Tasker and Herman, 2011). Multiple lines of research indicate that the endocannabinoid system of the BLA is involved in neural plasticity mechanisms related to emotional learning and memory (Akirav, 2011; Campolongo et al, 2009; Marsicano et al, 2002). As GR blockade did not attenuate the memoryenhancing effect of the cannabinoid agonist WIN55,212-2, our findings strongly suggest that the endocannabinoid pathway is a primary route by which glucocorticoids induce memory enhancement and, at least from a functional perspective, is located downstream from the GR site of action. Prior evidence that corticosterone administration rapidly ie, within $10 \mathrm{~min}$, elevates endocannabinoid levels in the amygdala (Hill et al, 2010a) supports this conclusion and further reinforces the nongenomic nature of this effect. On a broader level, these findings are consistent with evidence from in vitro studies, indicating that glucocorticoids rapidly inhibit parvocellular neurons in hypothalamic regions, in controlling hypothalamo-pituitary-adrenocortical axis activity, through a mechanism that involves the activation of a membrane-bound GR and synthesis of endocannabinoid ligands (Di et al, 2003).

Glucocorticoids selectively enhance the consolidation of emotionally arousing experiences because of a critical dependence on endogenous noradrenergic activation within the BLA (Buchanan and Lovallo, 2001; Kuhlmann and Wolf, 2006; Roozendaal et al, 2006a; Van Stegeren et al, 2007). In vitro electrophysiological findings support the conclu- 
sion that glucocorticoids interact with the noradrenergic system in altering plasticity and cellular activity in BLA neurons (Joëls et al, 2011). Previously, we reported that the $\beta$-adrenoceptor antagonist propranolol administered into the BLA blocked glucocorticoid effects on memory consolidation (Roozendaal et al, 2006a, 2006b). Our present finding that propranolol also blocked the WIN55,212-2 effect on memory indicates that endocannabinoids, like glucocorticoids, enhance the consolidation of memory via an interaction with the noradrenergic arousal system (Campolongo et al, 2013). We proposed in prior studies that the endocannabinoid system is a likely candidate for mediating the rapid effects of glucocorticoids onto stimulating the release of norepinephrine from presynaptic sites (Campolongo et al, 2009). Our present findings indicate that the endocannabinoid system also mediates glucocorticoid effects at the postsynaptic level on altering the sensitivity of the BLA to the memory-enhancing effects of noradrenergic stimulation. GR blockade is known to reduce the sensitivity of the BLA to the memory-enhancing effects of the $\beta$ adrenoceptor agonist clenbuterol such that a 100-times higher dose is required to induce memory enhancement (Roozendaal et al, 2002). We found here that a blockade of CB1 receptors induced an identical shift in the doseresponse effects of clenbuterol. Moreover, pharmacological augmentation of endocannabinoid signaling with an otherwise noneffective dose of WIN55,212-2 was sufficient to fully compensate for the GR antagonist effect and normalized the sensitivity of the BLA to the memory-enhancing effects of clenbuterol. These findings provide evidence for the view that recruitment of downstream endocannabinoid signaling is an essential intermediate step in mediating the permissive influence of glucocorticoids onto the noradrenergic system. The endocannabinoid system also mediates glucocorticoid effects onto the CRF system. It is well established that the glucocorticoid and CRF systems are intimately linked (Sawchenko, 1987). We previously reported that a GR antagonist infused into the BLA blocked the enhancing effect of CRF on memory consolidation (Roozendaal et al, 2008). Here, we demonstrate that CB1 receptor blockade also abolished CRF-induced memory enhancement. Moreover, a low dose of WIN55,212-2 compensated for the lack of glucocorticoid activity and reinstated the CRF-induced enhancement of memory. Although these findings imply local interactions of CRF with glucocorticoids and endocannabinoids within the BLA (Roozendaal et al, 2008), the possibility that CRF-induced memory enhancement is mediated through influences on the hypothalamo-pituitary-adrenocortical axis can presently not be excluded. At the physiological level, our findings suggest that GR signaling rapidly boosts the mobilization of endocannabinoids, which then increases the excitability of BLA pyramidal neurons, rendering them more sensitive to the memory-enhancing effects of both norepinephrine and CRF (Roozendaal et al, 2006b, 2008).

How can endocannabinoid signaling regulate the excitability of BLA neurons? CB1 receptor-mediated retrograde signaling can suppress the presynaptic release of both glutamate and GABA, inducing long-term changes in neuronal excitability. Within the $\mathrm{BLA}, \mathrm{CB} 1$ receptors are found predominantly on local inhibitory GABAergic interneurons. $A$ recent study indicated that $\mathrm{CB} 1$ receptors are particularly enriched in axon terminals of cholecystokinin (CCK)positive interneurons that form perisomatic synapses with pyramidal neurons in the BLA (Yoshida et al, 2011). CCK interneurons are proposed to function as fine-tuning devices for the cooperation of pyramidal neurons, and are sensitive to the emotional and motivational state of the animal (Katona et al, 2001; Yoshida et al, 2011). It has been reported that glucocorticoid administration rapidly elevates anandamide, but not 2-AG, levels in the amygdala (Hill et al, 2010a). Anandamide might trigger long-term depression at inhibitory synapses and decrease GABAergic transmission (Azad et al, 2004; Gunduz-Cinar et al, 2013). Such a suppression of inhibitory transmission can then increase the firing rate or excitability of BLA neurons through feedforward inhibition (Pistis et al, 2004). Interestingly, it has been reported that corticosterone also increases the spontaneous firing rate and excitability of principal BLA neurons via an influence on GABAergic circuits (Duvarci and Paré, 2007; Kavushansky and Richter-Levin, 2006). Extensive evidence indicates that an inhibition of local GABAergic circuits in the BLA enhances memory consolidation (Campolongo et al, 2009; Introini-Collison et al, 1994). A recent study reported that endocannabinoids can also alter BLA activity through an inhibition of CCK transmission (Bowers and Ressler, 2014), but whether this has a role in mediating glucocorticoid effects on memory remains to be investigated.

Collectively, our findings indicate that the endocannabinoid system is a primary route by which glucocorticoids

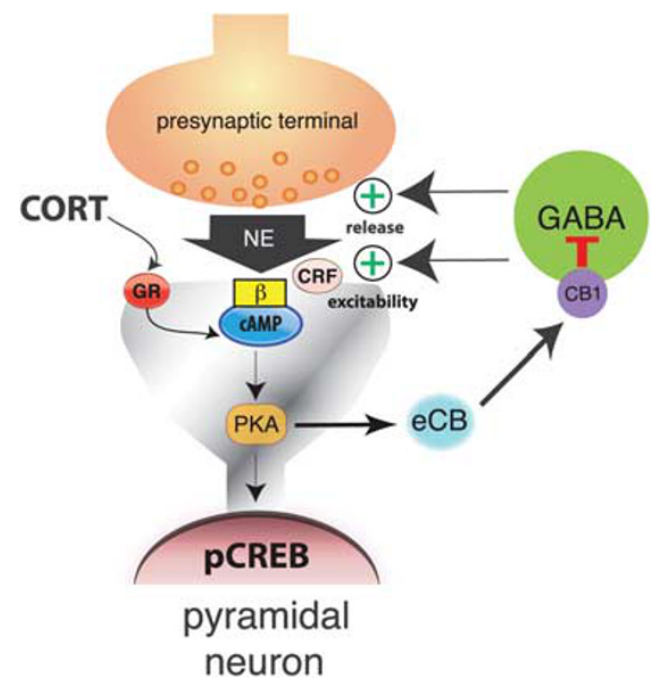

Figure 5 A model illustrating the role of the endocannabinoid system within the BLA in integrating the effects of glucocorticoids and the norepinephrine and CRF systems on memory consolidation. Corticosterone (CORT) is released during emotionally arousing events and binds to a membrane-bound GR, which activates the intracellular cAMP/PKA signaling pathway to induce endocannabinoid (eCB) synthesis. Endocannabinoids are then released into the synapse where they bind to $C B I$ receptors on GABAergic terminals and thereby inhibit the release of GABA. This suppression of GABA release subsequently disinhibits norepinephrine (NE) release, and increases the excitability of BLA pyramidal neurons, rendering them more sensitive to the effects of norepinephrine and CRF. Together, these effects will result in an increased activation of the CAMP/PKA pathway and phosphorylation of CAMP response element binding (CREB) protein. These stress hormone effects in the BLA are required for enhancing memory consolidation of emotionally arousing experiences. 
enhance memory consolidation and sensitize BLA neurons to the memory-enhancing effects of norepinephrine and CRF (Figure 5). They support the hypothesis that the endocannabinoid system acts to integrate the fine-tuned crosstalk between multiple stress hormone systems in the coordination of stress and emotional arousal effects on neural plasticity and memory consolidation. Although emotional enhancement of memory has obvious adaptive value in evolutionary terms, the present findings might also carry important implications for maladapative stress responses. Changes in glucocorticoid (Yehuda, 2009), endocannabinoid (Hauer et al, 2013; Hill et al, 2013; Neumeister et al, 2013), and catecholaminergic (Krystal and Neumeister, 2009) signaling have repeatedly been demonstrated in individuals after malignant stress exposure and the development of posttraumatic stress disorder (PTSD). Critically, a recent study reported that the combined assessment of alterations in cortisol and anandamide levels has a much higher predictive value in classifying PTSD cases than analysis of these factors individually (Neumeister et al, 2013). These findings underscore the importance of delineating the complex interactions between the multiple stress hormone systems in the regulation of memory consolidation of emotionally arousing, and sometimes traumatic, experiences.

\section{FUNDING AND DISCLOSURE}

The authors declare no conflict of interest. This study was supported by the Else-Kröner-Fresenius-Foundation, Germany (to D.H.).

\section{ACKNOWLEDGEMENTS}

We thank Dr Tansu Celikel for providing lab facilities, Elisabeth Pierson, Department of General Instruments, for help with the acquisition of confocal images and $\mathrm{Dr}$ Matthew Hill for critical comments on the manuscript.

\section{REFERENCES}

Akirav I (2011). The role of cannabinoids in modulating emotional and non-emotional memory processes in the hippocampus. Front Behav Neurosci 5: 34.

Atsak P, Hauer D, Campolongo P, Schelling G, McGaugh JL, Roozendaal B (2012a). Glucocorticoids interact with the hippocampal endocannabinoid system in impairing retrieval of contextual fear memory. Proc Natl Acad Sci USA 109: 3504-3509.

Atsak P, Roozendaal B, Campolongo P (2012b). Role of the endocannabinoid system in regulating glucocorticoid effects on memory for emotional experiences. Neuroscience 204: 104-116.

Azad SC, Monory K, Marsicano G, Cravatt BF, Lutz B, Zieglgansberger $\mathrm{W}$ et al (2004). Circuitry for associative plasticity in the amygdala involves endocannabinoid signaling. J Neurosci 24: 9953-9961.

Barsegyan A, Mackenzie SM, Kurose BD, McGaugh JL, Roozendaal B (2010). Glucocorticoids in the prefrontal cortex enhance memory consolidation and impair working memory by a common neural mechanism. Proc Natl Acad Sci USA 107: 16655-16660.

Bowers ME, Ressler KJ (2014). Interaction between the cholecystokinin and endogenous cannabinoid systems in cued fear expression and extinction retention. Neuropsychopharmacology.

Buchanan TW, Lovallo WR (2001). Enhanced memory for emotional material following stress-level cortisol treatment in humans. Psychoneuroendocrinology 26: 307-317.
Campolongo P, Morena M, Scaccianoce S, Trezza V, Chiarotti F, Schelling G et al (2013). Novelty-induced emotional arousal modulates cannabinoid effects on recognition memory and adrenocortical activity. Neuropsychopharmacology 38: 1276-1286.

Campolongo P, Roozendaal B, Trezza V, Hauer D, Schelling G, McGaugh JL et al (2009). Endocannabinoids in the rat basolateral amygdala enhance memory consolidation and enable glucocorticoid modulation of memory. Proc Natl Acad Sci USA 106: $4888-4893$.

Christianson SA (1992). Emotional stress and eyewitness memory: a critical review. Psychol Bull 112: 284-309.

Dallman M (2005). Fast glucocorticoid actions on brain: back to the future. Front Neuroendocrinol 26: 103-108.

de Kloet ER, Oitzl MS, Joëls M (1999). Stress and cognition: are corticosteroids good or bad guys? Trends Neurosci 22: 422-426.

de Quervain DJ, Aerni A, Schelling G, Roozendaal B (2009). Glucocorticoids and the regulation of memory in health and disease. Front Neuroendocrinol 30: 358-370.

Di S, Malcher-Lopes R, Halmos KC, Tasker JG (2003). Nongenomic glucocorticoid inhibition via endocannabinoid release in the hypothalamus: a fast feedback mechanism. J Neurosci 23: 4850-4857.

Duvarci S, Paré D (2007). Glucocorticoids enhance the excitability of principal basolateral amygdala neurons. I Neurosci 27: 4482-4491.

Evanson NK, Tasker JG, Hill MN, Hillard CJ, Herman JP (2010). Fast feedback inhibition of the HPA axis by glucocorticoids is mediated by endocannabinoid signaling. Endocrinology 151: 4811-4819.

Fornari RV, Wichmann R, Atsak P, Atucha E, Barsegyan A, Beldjoud $\mathrm{H}$ et al (2012). Rodent stereotaxic surgery and animal welfare outcome improvements for behavioral neuroscience. J Vis Exp e3528.

Gunduz-Cinar O, MacPherson KP, Cinar R, Gamble-George J, Sugden K, Williams B et al (2013). Convergent translational evidence of a role for anandamide in amygdala-mediated fear extinction, threat processing and stress-reactivity. Mol Psychiatry 18: 813-823.

Hauer D, Schelling G, Gola H, Campolongo P, Morath J, Roozendaal B et al (2013). Plasma concentrations of endocannabinoids and related primary fatty acid amides in patients with post-traumatic stress disorder. PLoS One 8: e62741.

Hill M, Karatsoreos I, Hillard C, Mcewen B (2010a). Rapid elevations in limbic endocannabinoid content by glucocorticoid hormones in vivo. Psychoneuroendocrinology 35: 1333-1338.

Hill M, McEwen B (2010b). Involvement of the endocannabinoid system in the neurobehavioural effects of stress and glucocorticoids. Prog Neuropsychopharmacol Biol Psychiatry 34: 791-797.

Hill M, Patel S, Campolongo P, Tasker JG, Wotjak CT, Bains JS (2010c). Functional interactions between stress and the endocannabinoid system: from synaptic signaling to behavioral output. J Neurosci 30: 14980-14986.

Hill MN, Bierer LM, Makotkine I, Golier JA, Galea S, McEwen BS et al (2013). Reductions in circulating endocannabinoid levels in individuals with post-traumatic stress disorder following exposure to the world trade center attacks. Psychoneuroendocrinology 38: 2952-2961.

Introini-Collison IB, Castellano C, McGaugh JL (1994). Interaction of GABAergic and beta-noradrenergic drugs in the regulation of memory storage. Behav Neural Biol 61: 150-155.

Joëls M, Fernandez G, Roozendaal B (2011). Stress and emotional memory: a matter of timing. Trends Cogn Sci 15: 280-288.

Johnson L, Farb C, Morrison J, McEwen B, Ledoux J (2005). Localization of glucocorticoid receptors at postsynaptic membranes in the lateral amygdala. Neuroscience 136: 289-299.

Josselyn SA, Kida S, Silva AJ (2004). Inducible repression of CREB function disrupts amygdala-dependent memory. Neurobiol Learn Mem 82: 159-163. 
Kano M, Ohno-Shosaku T, Hashimotodani Y, Uchigashima M, Watanabe M (2009). Endocannabinoid-mediated control of synaptic transmission. Physiol Rev 89: 309-380.

Katona I, Rancz EA, Acsady L, Ledent C, Mackie K, Hajos N et al (2001). Distribution of CB1 cannabinoid receptors in the amygdala and their role in the control of GABAergic transmission. J Neurosci 21: 9506-9518.

Kavushansky A, Richter-Levin G (2006). Effects of stress and corticosterone on activity and plasticity in the amygdala. J Neurosci Res 84: 1580-1587.

Krystal JH, Neumeister A (2009). Noradrenergic and serotonergic mechanisms in the neurobiology of posttraumatic stress disorder and resilience. Brain Res 1293: 13-23.

Kuhlmann S, Wolf OT (2006). Arousal and cortisol interact in modulating memory consolidation in healthy young men. Behav Neurosci 120: 217-223.

Marsicano G, Wotjak CT, Azad SC, Bisogno T, Rammes G, Cascio MG et al (2002). The endogenous cannabinoid system controls extinction of aversive memories. Nature 418: 530-534.

McReynolds JR, Donowho K, Abdi A, McGaugh JL, Roozendaal B, McIntyre CK (2010). Memory-enhancing corticosterone treatment increases amygdala norepinephrine and Arc protein expression in hippocampal synaptic fractions. Neurobiol Learn Mem 93: 312-321.

Miranda MI, Quirarte GL, Rodriguez-Garcia G, McGaugh JL, Roozendaal B (2008). Glucocorticoids enhance taste aversion memory via actions in the insular cortex and basolateral amygdala. Learn Mem 15: 468-476.

Neumeister A, Normandin MD, Pietrzak RH, Piomelli D, Zheng MQ, Gujarro-Anton A et al (2013). Elevated brain cannabinoid CB1 receptor availability in post-traumatic stress disorder: a positron emission tomography study. Mol Psychiatry 18: 1034-1040.

Paxinos G, Watson C (2007). The Rat brain in stereotaxic Coordinates. 6th edn, Academic Press.

Pistis M, Perra S, Pillolla G, Melis M, Gessa GL, Muntoni AL (2004). Cannabinoids modulate neuronal firing in the rat basolateral amygdala: evidence for CB1- and non-CB1-mediated actions. Neuropharmacology 46: 115-125.

Roozendaal B, Hernandez A, Cabrera SM, Hagewoud R, Malvaez M, Stefanko DP et al (2010). Membrane-associated glucocorticoid activity is necessary for modulation of long-term memory via chromatin modification. J Neurosci 30: 5037-5046.

Roozendaal B, McGaugh JL (2011). Memory modulation. Behav Neurosci 125: 797-824.

Roozendaal B, Okuda S, de Quervain DJ, McGaugh JL (2006a). Glucocorticoids interact with emotion-induced noradrenergic activation in influencing different memory functions. Neuroscience 138: 901-910.

Roozendaal B, Okuda S, Van der Zee EA, McGaugh JL (2006b). Glucocorticoid enhancement of memory requires arousalinduced noradrenergic activation in the basolateral amygdala. Proc Natl Acad Sci USA 103: 6741-6746.

Roozendaal B, Quirarte GL, McGaugh JL (2002). Glucocorticoids interact with the basolateral amygdala beta-adrenoceptorcAMP/PKA system in influencing memory consolidation. Eur J Neurosci 15: 553-560.

Roozendaal B, Schelling G, McGaugh JL (2008). Corticotropinreleasing factor in the basolateral amygdala enhances memory consolidation via an interaction with the beta-adrenoceptorcAMP pathway: dependence on glucocorticoid receptor activation. J Neurosci 28: 6642-6651.

Salehi B, Cordero MI, Sandi C (2010). Learning under stress: the inverted-U-shape function revisited. Learn Mem 17: 522-530.

Sawchenko PE (1987). Evidence for a local site of action for glucocorticoids in inhibiting CRF and vasopressin expression in the paraventricular nucleus. Brain Res 403: 213-223.

Tasker JG, Herman JP (2011). Mechanisms of rapid glucocorticoid feedback inhibition of the hypothalamic-pituitary-adrenal axis. Stress 14: 398-406.

Van Stegeren A, Wolf OT, Everaerd W, Scheltens P, Barkhof F, Rombouts S (2007). Endogenous cortisol level interacts with noradrenergic activation in the human amygdala. Neurobiol Learn Mem 87: 57-66.

Yehuda R (2009). Status of glucocorticoid alterations in posttraumatic stress disorder. Ann NY Acad Sci 1179: 56-69.

Yoshida T, Uchigashima M, Yamasaki M, Katona I, Yamazaki M, Sakimura $\mathrm{K}$ et al (2011). Unique inhibitory synapse with particularly rich endocannabinoid signaling machinery on pyramidal neurons in basal amygdaloid nucleus. Proc Natl Acad Sci USA 108: 3059-3064.

Supplementary Information accompanies the paper on the Neuropsychopharmacology website (http://www.nature.com/npp) 